\title{
BIFURCATION IN ELASTIC-PLASTIC SOLIDS IN PLANE STRESS*
}

\author{
BY \\ R. N. DUBEY AND S. T. ARIARATNAM \\ University of Waterloo
}

Summary. A sufficient condition for bifurcation of equilibrium for an elasticplastic solid under a state of plane stress is established. The bifurcation is found to occur in any of the following modes: (i) symmetric mode corresponding to necking or bulging, (ii) antisymmetric mode corresponding to buckling and (iii) mode of deformation localized at the surface.

Introduction. The stability and bifurcation of rigid-plastic solids in plane-plastic flow has been studied extensively [1-3]. Similar work for elastic-plastic solids in plane flow is also available [4]. However, very little work seems to have been done on the problems of stability and bifurcation of an elastic-plastic solid in three-dimensional flow. A similar investigation for rigid-plastic solids in three-dimensional flow is limited in scope due to the restricted choice of the velocity field.

Prager [5] found that for a von Mises solid under a state of uniform stress, the only admissible velocity field in three-dimensional flow is of quadratic form. Hence some form of instability may be excluded when the behaviour of the solid is postulated to be rigid plastic (example-surface instabilities, buckling). In the degenerate case for a von Mises solid, when one principal component of the stress deviation is zero as in plane strain, or for a Tresca solid, a transcendental form of the velocity field is admissible. Even then it would appear that a rigid-plastic solid does not exhibit instability localized at the surface.

In this analysis, an elastic-plastic plate in a state of plane stress is considered. In the constitutive equation, which is due to Hill [6], the plastic component of deformation is expressed in terms of the components of the unit normal to the yield surface at the local stress point. Hence the analysis is applicable to a wide class of inelastic solids.

As an illustration, we consider a plate that is under equal biaxial stress and obeys von Mises' yield criterion. This assumption results in considerable mathematical simplification. It is found that the plate may become unstable in any of the following modes: (i) symmetric mode, (ii) antisymmetric mode or buckling mode, and (iii) instability localized at the surface.

Formulation of the problem. Consider an incompressible isotropic elastic-plastic disk in a state of plane stress at a certain time $t=0$ during a process of continued deformation. In the current configuration, also taken as reference configuration for incremental deformation, the plate is of in-plane dimension $2 a \times 2 b$ and of thickness $2 c$.

A fixed coordinate frame $x_{i}$ coinciding with the axes of symmetry of the plate is taken as the reference frame. Wherever convenient the coordinates $x_{1}, x_{2}, x_{3}$ will

*Received September 28, 1968; revised version received November 2, 1968. The research for this paper was supported in part by Grants Nos. A-1815 and A-5164 from the National Research Council of Canada. 
be replaced by $x, y, z$, and the velocity components $v_{1}, v_{2}, v_{3}$ by $u, v, w$, respectively. With respect to this frame, the current stress field, assumed homogeneous, is given by

$$
\sigma_{i i}=\left[\begin{array}{ccc}
\sigma_{11} & 0 & 0 \\
0 & \sigma_{22} & 0 \\
0 & 0 & 0
\end{array}\right] .
$$

In the current configuration, the components of the true stress $\sigma_{i j}$ and the nominal stress $s_{i j}$ are identical (Hill [6]).

We now investigate the condition for bifurcation of equilibrium of the body under continuing deformation. During the subsequent infinitesimal deformation, the faces $x_{3}= \pm c$ of the plate are given to be free of external traction-rate whereas the edges $x_{1}= \pm a, x_{2}= \pm b$, assumed to be frictionless, are prescribed to move with velocities $U$ and $V$, respectively. For conciseness, the traction-rate boundary condition is expressed in terms of the material derivative, that is the time derivative following the element, of the nominal stress, $\dot{s}_{i j}$. Hence,

$$
\begin{array}{lll}
\dot{s}_{12}=\dot{s}_{13}=0, \quad v_{i}=n_{i} U & \text { on the face } & x_{1}= \pm a, \\
\dot{s}_{21}=0 \dot{s}_{23}=0, \quad v_{i}=n_{i} V & \text { on the face } & x_{2}= \pm b, \\
\dot{s}_{3 i}=0, \quad i=1,2,3 & \text { on the face } & x_{3}= \pm c,
\end{array}
$$

where $n_{i}$ is the unit outward normal to the boundary surface.

The incremental stress field must satisfy the equations of continuing equilibrium,

$$
\dot{s}_{i j, i}=0 \text {. }
$$

Material properties. We consider the Jaumann derivative of the true stress $D \sigma^{i i} / D t$ as the objective stress-rate for describing the material property. The fixed components of $D \sigma^{i i} / D t$ are given by the relation [7]

$$
D \sigma^{i i} / D t=\dot{\sigma}^{i j}+\sigma^{i k} \omega_{k}^{i}+\sigma^{i k} \omega_{k}^{i},
$$

where $\omega_{i j}=\frac{1}{2}\left(v_{i, j}-v_{i, i}\right)$ is the antisymmetric part of the velocity gradient tensor. The constitutive equation for an incompressible and isotropic elastic-plastic solid may be taken in the form

$$
D \sigma_{i j} / D t=2 \mu\left(\epsilon_{i j}-\epsilon_{i j}^{(p)}\right)+\dot{p} \delta_{i i}
$$

where

$$
\begin{aligned}
& \epsilon_{i j}^{(p)}=h^{-1} m_{i j}\left(m_{k l} D \sigma_{i j} / D t\right) \text { when } m_{i j} D \sigma_{i j} / D t>0 \text {, } \\
& =\begin{array}{lll}
0 & \ldots & \leq 0
\end{array}
\end{aligned}
$$

Here $\epsilon_{i j}$ is the strain-rate tensor, $\epsilon_{i j}^{(p)}$ the plastic part of the strain rate, $m_{i j}$ the typical component of the unit normal to the yield surface at the local stress point, $h$ the positive scalar measure of the current rate of work hardening, $\mu$ Lamés constant, and $\dot{p}$ the hydrostatic pressure rate.

In view of the current stress distribution (1), the components of $m_{i j}$ may be taken in the form: 


$$
m_{i j}=\left[\begin{array}{ccc}
m_{11} & 0 & 0 \\
0 & m_{22} & 0 \\
0 & 0 & m_{33}
\end{array}\right],
$$

where

$$
m_{i i}=0, \text { and } \quad m_{i j} m_{i j}=1 .
$$

With the use of (1), (5), (7) and (8), the relation (6), for material undergoing plastic loading everywhere, can be written:

$$
\begin{aligned}
& \dot{\sigma}_{11}=2 \mu\left(a_{11} u_{x}+a_{12} v_{y}+a_{13} w_{z}\right)+\dot{p}, \\
& \dot{\sigma}_{22}=2 \mu\left(a_{12} u_{x}+a_{22} v_{y}+a_{23} w_{z}\right)+\dot{p}, \\
& \dot{\sigma}_{33}=2 \mu\left(a_{13} u_{x}+a_{23} v_{y}+a_{33} w_{z}\right)+\dot{p}, \\
& \dot{\sigma}_{12}=2 \mu\left(a_{41} u_{y}+a_{42} v_{x}\right), \\
& \dot{\sigma}_{23}=2 \mu\left(a_{51} v_{z}+a_{52} w_{y}\right), \\
& \dot{\sigma}_{13}=2 \mu\left(a_{61} w_{x}+a_{62} u_{z}\right),
\end{aligned}
$$

where

$$
\begin{array}{rlrl}
a_{11} & =1-m_{11}^{2} \delta /(1+\delta), & a_{22} & =1-m_{22}^{2} \delta /(1+\delta), \\
a_{33} & =1-m_{33}^{2} \delta /(1+\delta), & a_{12} & =-m_{11} m_{22} \delta /(1+\delta), \\
a_{23} & =-m_{22} m_{33} \delta /(1+\delta), & a_{13} & =-m_{11} m_{33} \delta /(1+\delta), \\
a_{41} & =\frac{1}{2}\left[1-\left(\theta_{1}-\theta_{2}\right)\right], & a_{42} & =\frac{1}{2}\left[1+\left(\theta_{1}-\theta_{2}\right)\right], \\
a_{51} & =\frac{1}{2}\left[1-\theta_{2}\right], & a_{52} & =\frac{1}{2}\left[1+\theta_{2}\right], \\
a_{61} & =\frac{1}{2}\left[1+\theta_{1}\right], & a_{62} & =\frac{1}{2}\left[1-\theta_{1}\right], \\
& \theta_{1}=\left(\sigma_{11} / 2 \mu\right), & \theta_{22}=\left(\sigma_{2} / 2 \mu\right), \quad \delta=(2 \mu / h),
\end{array}
$$

and the latin suffixes denote partial differentiation.

Homogeneous deformation. The relation (Hill [6])

$$
\dot{s}_{i j}=\dot{\sigma}_{i j}-\sigma_{i k} v_{i, k}
$$

and the incompressibility condition

$$
v_{i, i}=0
$$

can be used to express the equations of equilibrium (3) in the form

$$
\dot{\sigma}_{i, i}=0 \text {. }
$$

A homogeneous deformation field satisfying (3a) and the boundary condition (2) is found by inspection to be

$$
u=U x / a, \quad v=V y / b, \text { and } w=-(U / a+V / b) z .
$$

The deformation field (13) preserves the rectangular shape of the plate. The associated 
stress-rate field is, using (2), (9), (10) and (13),

$$
\begin{aligned}
& \dot{\sigma}_{11}=2 \mu\left[2-\left(m_{11}-m_{33}\right)^{2} \frac{\delta}{1+\delta}\right] U / a+2 \mu\left[1-\left(m_{11} m_{22}+2 m_{33}^{2}\right) \frac{\delta}{1+\delta}\right] V / b, \\
& \dot{\sigma}_{22}=2 \mu\left[1-\left(m_{11} m_{22}+2 m_{33}^{2}\right) \frac{\delta}{1+\delta}\right] U / a+2 \mu\left[2-\left(m_{22}-m_{33}\right)^{2} \frac{\delta}{1+\delta}\right] V / b, \\
& \dot{\sigma}_{33}=\dot{\sigma}_{12}=\dot{\sigma}_{13}=\dot{\sigma}_{23}=0 .
\end{aligned}
$$

Now we look for a velocity field, not identically zero, but such that its components $u$ and $v$ vanish on the edges $x= \pm a$ and $y= \pm b$ respectively and which satisfies the equations of continuing equilibrium ( $3 \mathrm{a})$ and the traction-rate boundary conditions (2). It is obvious that the nonhomogeneous deformation field, when multiplied by an arbitrary constant and added to the homogeneous field (13) will still satisfy the equations (2) and (3). Thus there is a bifurcation of equilibrium in the current state.

It may be mentioned that the in-plane dimension of the plate is not altered by the nonhomogeneous field.

Nonhomogeneous deformation. The velocity component $w$ and the pressure rate $\dot{p}$ can be eliminated from (3a) by using (9), (10) and (11), to yield

$$
\begin{gathered}
{\left[1-2\left(2 m_{11}^{2}+m_{22} m_{33}\right) \delta /(1+\delta)\right] u_{x x y}+\left[1-\left(\theta_{1}-\theta_{2}\right)\right] u_{y y y}+\left(1-\theta_{1}\right) v_{y z z}} \\
-\left[1+\left(\theta_{1}-\theta_{2}\right) v_{x x x}-\left[1-2\left(2 m_{22}^{2}+m_{11} m_{33}\right) \delta /(1+\delta)\right] v_{x y y}\right. \\
-\left(1-\theta_{2}\right) v_{x z z}=0 \\
\left(1+\theta_{1}\right) u_{x x x x}+\left(1+\theta_{2}\right) u_{x x y y}+2\left[1-\left(m_{11}-m_{33}\right)^{2} \delta /(1+\delta)\right] u_{x x z z} \\
+\left[1-\left(\theta_{1}-\theta_{2}\right)\right] u_{y y z z}+\left(1-\theta_{1}\right) u_{z z z z}+\left(1+\theta_{1}\right) v_{x x x y}+\left(1+\theta_{2}\right) v_{x y y y} \\
+\left[1-2\left(2 m_{33}^{2}+m_{11} m_{22}\right) \delta /(1+\delta)\right] v_{x y z z}=0 .
\end{gathered}
$$

We seek a solution for $v_{i}$ in (15) in the form

$$
u=\alpha_{1} \sin \nu x \cos \eta y \exp (\beta z), \quad v=\alpha_{2} \cos \nu x \sin \eta y \exp (\beta z),
$$

where $\alpha_{1}, \alpha_{2}$ are constants.

A necessary and sufficient condition for a nontrivial solution of $\alpha_{1}$ and $\alpha_{2}$ to exist is found by substituting for $u$ and $v$ in (15) and equating the determinant of the coefficients of $\alpha_{1}$ and $\alpha_{2}$ to zero. This condition is obtained in the form

$$
\begin{aligned}
\beta^{6}\left(1-\theta_{1}\right) & \left(1-\theta_{2}\right) \\
& -\beta^{4}\left(1-\theta_{1}\right)\left[\left\{1+\left(\theta_{1}-\theta_{2}\right)\right\} \nu^{2}+2\left\{1-\left(m_{22}-m_{33}\right)^{2} \delta /(1+\delta)\right\} \eta^{2}\right] \\
& +\left(1-\theta_{2}\right)\left[2\left\{1-\left(m_{11}-m_{33}\right)^{2} \delta(1+\delta)\right\} \nu^{2}+\left\{1-\left(\theta_{1}-\theta_{2}\right)\right\} \eta^{2}\right] \\
& +\beta^{2}\left\{\left[1+\left(\theta_{1}-\theta_{2}\right)\right]\left[3-2\left(m_{11}-m_{33}\right)^{2} \delta /(1+\delta)\right] \nu^{4}\right. \\
& +\left[1-\left(\theta_{1}-\theta_{2}\right)\right]\left[3-2\left(m_{22}-m_{33}\right)^{2} \delta /(1+\delta)\right] \eta^{4}-\theta_{1} \theta_{2}\left(\nu^{2}-\eta^{2}\right)^{2} \\
& \left.+\nu^{2} \eta^{2}\left[6 /(1+\delta)-2\left(\theta_{1}^{2}+\theta_{2}^{2}\right)\right]\right\} \\
& -\left[\left(1+\theta_{1}\right) \nu^{2}+\left(1+\theta_{2}\right) \eta^{2}\right]\left\{\left[1+\left(\theta_{1}-\theta_{2}\right)\right] \nu^{4}\right. \\
& \left.+2\left[1-\left(m_{11}-m_{22}\right)^{2} \delta /(1+\delta)\right] \nu^{2} \eta^{2}+\left[1-\left(\theta_{1}-\theta_{2}\right)\right] \eta^{4}\right\}=0 .
\end{aligned}
$$


Once the values of the roots $\beta_{i}(i=1, \cdots, 6)$ of Eq. (17) are determined, the ratio $\left(\alpha_{2} / \alpha_{1}\right)$ can then be obtained from (15). Thus $u$ and $v$ are known except for an arbitrary constant multiplier, and $w$ is found from the incompressibility condition (12). The condition for bifurcation of equilibrium can now be obtained in a routine manner.

The actual field $v_{i}$ is equal to the sum of the homogeneous field (13) and the nonhomogeneous field multiplied by an arbitrary constant, which can be chosen so that the material everywhere loads during the deformation. This procedure results in considerable mathematical simplification.

As an illustration, we consider a body under equal all-round tension and obeying von Mises' yield criterion. Thus $\sigma_{11}=\sigma_{22}=\sigma$ (say), and $m_{11}=1 / \sqrt{ } 6, m_{22}=1 / \sqrt{ } 6$, $m_{33}=-2 / \sqrt{ } 6$. The characteristic equation (17) now reduces to

$\beta^{\theta}(1-\theta)^{2}+3 \beta^{4}(1-\theta)\left(\nu^{2}+\eta^{2}\right) /(1+\delta)+\beta^{2}\left(\nu^{2}+\eta^{2}\right)$

$$
\cdot\left[\frac{3}{1+\delta}-\theta^{2}\right]+\left(\nu^{2}+\eta^{2}\right)^{3}(1+\theta)=0
$$

where $\theta=\sigma / 2 \mu, \delta=2 \mu / h, \nu=m \pi / a, \eta=n \pi / b ; m$ and $n$ being positive integers.

The roots of (18) are

$$
\begin{aligned}
\beta^{2} & =\frac{\nu^{2}+\eta^{2}}{1-\theta}=\gamma^{2} \quad \text { (say) } \\
& =\gamma^{2} \delta_{1}^{2} \\
& =\gamma^{2} \delta_{2}^{2},
\end{aligned}
$$

where

$$
\begin{aligned}
& \delta_{1}^{2}=\frac{2-\delta}{2(1+\delta)}+\left[\theta^{2}-\frac{3 \delta(\delta+4)}{4(1+\delta)^{2}}\right]^{1 / 2}, \text { and } \\
& \delta_{2}^{2}=\frac{2-\delta}{2(1+\delta)}-\left[\theta^{2}-\frac{3 \delta(\delta+4)}{4(1+\delta)^{2}}\right]^{1 / 2} .
\end{aligned}
$$

Hence, from (15)

$$
\begin{aligned}
u= & \sin \nu x \cos \eta y\left[\alpha_{1} \exp (\gamma z)+\alpha_{2} \exp (-\gamma z)+\alpha_{3} \exp \left(\gamma \delta_{1} z\right)\right. \\
& \left.+\alpha_{4} \exp \left(-\gamma \delta_{1} z\right)+\alpha_{5} \exp \left(\gamma \delta_{2} z\right)+\alpha_{6} \exp \left(-\gamma \delta_{2} z\right)\right], \\
v= & \cos \nu x \sin \eta y\left\{(-\nu / \eta)\left[\alpha_{1} \exp (\gamma z)+\alpha_{2} \exp (-\gamma z)\right]\right. \\
& \left.+(\eta / \nu)\left[\alpha_{3} \exp \left(\gamma \delta_{1} z\right)+\alpha_{4} \exp \left(-\gamma \delta_{1} z\right)+\alpha_{5} \exp \left(\gamma \delta_{2} z\right)+\alpha_{6} \exp \left(-\gamma \delta_{2} z\right)\right]\right\}, \\
w= & -\gamma[(1-\theta) / \nu] \cos \nu x \cos \eta y\left[\left(\alpha_{3} / \delta_{1}\right) \exp \left(\gamma \delta_{1} z\right)\right. \\
& \left.-\left(\alpha_{4} / \delta_{1}\right) \exp \left(-\gamma \delta_{1} z\right)+\left(\alpha_{5} / \delta_{2}\right) \exp \left(\gamma \delta_{2} z\right)-\left(\alpha_{6} / \delta_{2}\right) \exp \left(-\gamma \delta_{2} z\right)\right] .
\end{aligned}
$$

Bifurcation may occur in any of the following modes: (i) symmetric mode of deformation corresponding to the necking or bulging of the specimen, (ii) antisymmetric mode of deformation corresponding to buckling mode, (iii) a mode of deformation localized at the surface (surface instability). The three cases are considered separately.

Symmetric mode of deformation. The velocity field in this case is symmetrical about the $x-y$ plane; consequently $u(z)=u(-z), v(z)=v(-z), w(z)=-w(-z)$. 
Only even functions of $z$ need therefore be considered in the expressions for $u$ and $v$ which may then be taken in the form:

$$
\begin{aligned}
& u=\sin \nu x \cos \eta y\left[\alpha_{1} \cosh \gamma z+\alpha_{2} \cosh \gamma \delta_{1} z+\alpha_{3} \cosh \gamma \delta_{2} z\right], \\
& v=\cos \nu x \sin \eta y\left[-\alpha_{1}(\nu / \eta) \cosh \gamma_{2}+(\eta / \nu)\left(\alpha_{2} \cosh \gamma \delta_{1} z+\alpha_{3} \cosh \gamma \delta_{2} z\right)\right],
\end{aligned}
$$

and

$$
w=-\gamma((1-\theta) / \nu) \cos \nu x \cos \eta y\left[\left(\alpha_{2} / \delta_{1}\right) \sinh \gamma \delta_{1} z+\left(\alpha_{3} / \delta_{2}\right) \sinh \gamma \delta_{2} z\right]
$$

follows by using (12).

The boundary condition (2c), on substituting from (9), (10), (11), (12) and (20), yields

$$
\begin{aligned}
\alpha_{1} \sinh \gamma c & +\alpha_{2}\left[\delta_{1}+(1-\theta) / \delta_{1}\right] \sinh \gamma \delta_{1} c+\alpha_{3}\left[\delta_{2}+(1-\theta) / \delta_{2}\right] \sinh \gamma \delta_{2} c=0, \\
-\alpha_{1} \sinh \gamma c+ & +\alpha_{2}(\eta / \nu)^{2}\left[\delta_{1}+(1-\theta) / \delta_{1}\right] \sinh \gamma \delta_{1} c \\
& \quad+\alpha_{3}(\eta / \nu)^{2}\left[\delta_{2}+(1-\theta) / \delta_{2}\right] \sinh \gamma \delta_{2} c=0, \\
& \alpha_{2}\left[3 /(1+\delta)-\theta-\delta_{1}^{2}\right] \cosh \gamma \delta_{1} c+\alpha_{3}\left[3 /(1+\delta)-\theta-\delta_{2}^{2}\right] \cosh \gamma \delta_{2} c=0 .
\end{aligned}
$$

A necessary and sufficient condition for bifurcation of equilibrium is obtained by eliminating $\alpha_{1}, \alpha_{2}$ and $\alpha_{3}$, and may be expressed in the form:

$$
\frac{\delta_{2} \tanh \gamma \delta_{1} c}{\delta_{1} \tanh \gamma \delta_{2} c}=\frac{\left[\frac{3}{1+\delta}-\theta-\delta_{1}^{2}\right]\left[1-\theta+\delta_{2}^{2}\right]}{\left[\frac{3}{1+\delta}-\theta-\delta_{2}^{2}\right]\left[1-\theta+\delta_{1}^{2}\right]} .
$$

The unknown in this equation is the critical stress $\sigma$. The least value of $\sigma$ obtained by minimizing with respect to $\nu$ and $\eta$ gives the first bifurcation state. For a thin plate, for which the ratio of the thickness to the smallest in-plane dimension of the plate is small, i.e., $c / a, c / b \ll 1$, the critical stress for the initiation of necking is obtained as

$$
\sigma=\mu \frac{4+\delta}{1+\delta}\left[1+\frac{\pi^{2} c^{2}}{3}\left(\frac{m^{2}}{a^{2}}+\frac{n^{2}}{b^{2}}\right)\right] .
$$

The minimum value of $\sigma$, obtained for $m=1, n=1$, is

$$
\sigma=\mu \frac{4+\delta}{1+\delta}\left[1+\frac{\pi^{2} c^{2}}{3 a^{2}}\left(1+a^{2} / b^{2}\right)\right], \quad \delta=2 \mu / h .
$$

The second term in the bracket represents the effect of shear stiffening.

In general, the roots of the characteristic equation (18) are complex. Eq. (21) may then be expressed in more convenient form in the following manner.

Let the roots of (18) be given by $\beta=r_{1} \pm \sqrt{ }-1 r_{2}$. Then, from (19a),

$$
2 r_{1}^{2}=(2-\delta) / 2(1+\delta)+\left(1-\theta^{2}\right)^{1 / 2}, \quad 2 r_{2}^{2}=-(2-\delta) / 2(1+\delta)+\left(1-\theta^{2}\right) .
$$

Proceeding as before, the condition for bifurcation of equilibrium is obtained from the boundary condition (2c), and may be written in the form $f_{1}(\theta)=f_{2}(\theta)$, where

$$
\begin{aligned}
& f_{1}=(1-\varphi) /(1+\varphi), \\
& f_{2}=\left(\frac{1+\theta}{1-\theta}\right)^{1 / 2}\left[1-\frac{4+\delta}{2 \theta(1+\delta)}\right], \quad \text { and } \\
& \varphi=\left(r_{1} \sin 2 \gamma r_{2} c\right) /\left(r_{2} \sinh 2 \gamma r_{1} c\right), \quad \delta=2 \mu / h .
\end{aligned}
$$


The curve of $f_{1}$ against $\theta$ for a given body constitutes a family, each corresponding to a different value of $\nu$ and $\eta$. The point of intersection of this family of curves with the curve of $f_{2}$ against $\theta$ corresponds to the critical value of $\theta$ at which bifurcation of equilibrium is to be expected. In Figure 1, the curves $f_{1}(\theta)$ and $f_{2}(\theta)$ against $\theta$ are shown for $m=1, n=1, a=b / 2$ and for $c / a=0.1,0.25$ and 1.0. It is seen that $f_{1}$ curves corresponding to $c / a=0.1$ and 0.25 each has only one point of intersection with $f_{2}$ curve. The corresponding mode of deformation is possible under tensile load. The $f_{1}$ curve corresponding to $c / a=1.0$ has two points of intersection with the $f_{2}$ curve; the point $C$ corresponds to necking mode (under tensile load) and the point $D$ corresponds to bulging mode (under compressive load). Similar curves can be drawn for other values of $m, n, a / b$ and $c / a$.

The two cases (a) $c / a, c / b \ll 1$, and (b) $c / a, c / b \gg$ are of particular interest and are considered in detail.

Case (a): $c / a, c / b \ll 1$. In this case $(\pi c / a)\left(m^{2}+n^{2} d^{2}\right)^{1 / 2} \ll 1, d=(a / b)$. Hence $\varphi$ tends to unity and consequently $f_{1}$ tends to zero. The line $f_{1}=0$, as is seen in Figure 2, does not intersect the family of $f_{2}$-curves for values of $\delta<2$; that is, the tensile instability of the type considered does not occur until the ratio $\delta$ exceeds 2 . The number of half-waves occurring at this instant, however, is indeterminate.

Case (b): $c / a, c / b \gg 1$. In this case $(m c / a)\left(m^{2}+n^{2} d^{2}\right)^{1 / 2} \gg 1$; consequently $\varphi$ tends to zero, and $f_{1}$ tends to unity. Under these conditions, the bifurcation of equilibrium is possible under both compressive and tensile loads. The number of half-waves is indeterminate in this case as well.

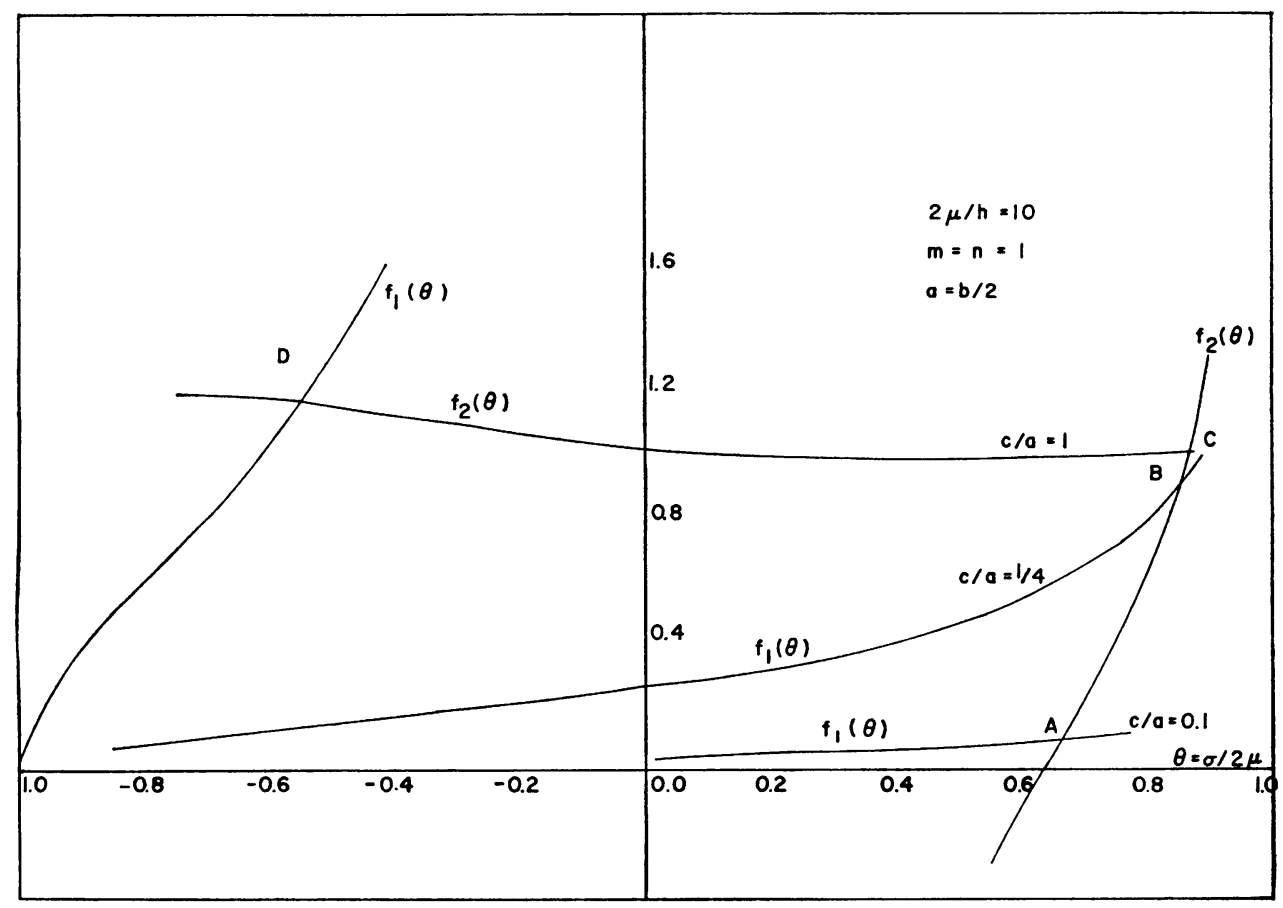

FIG. 1 


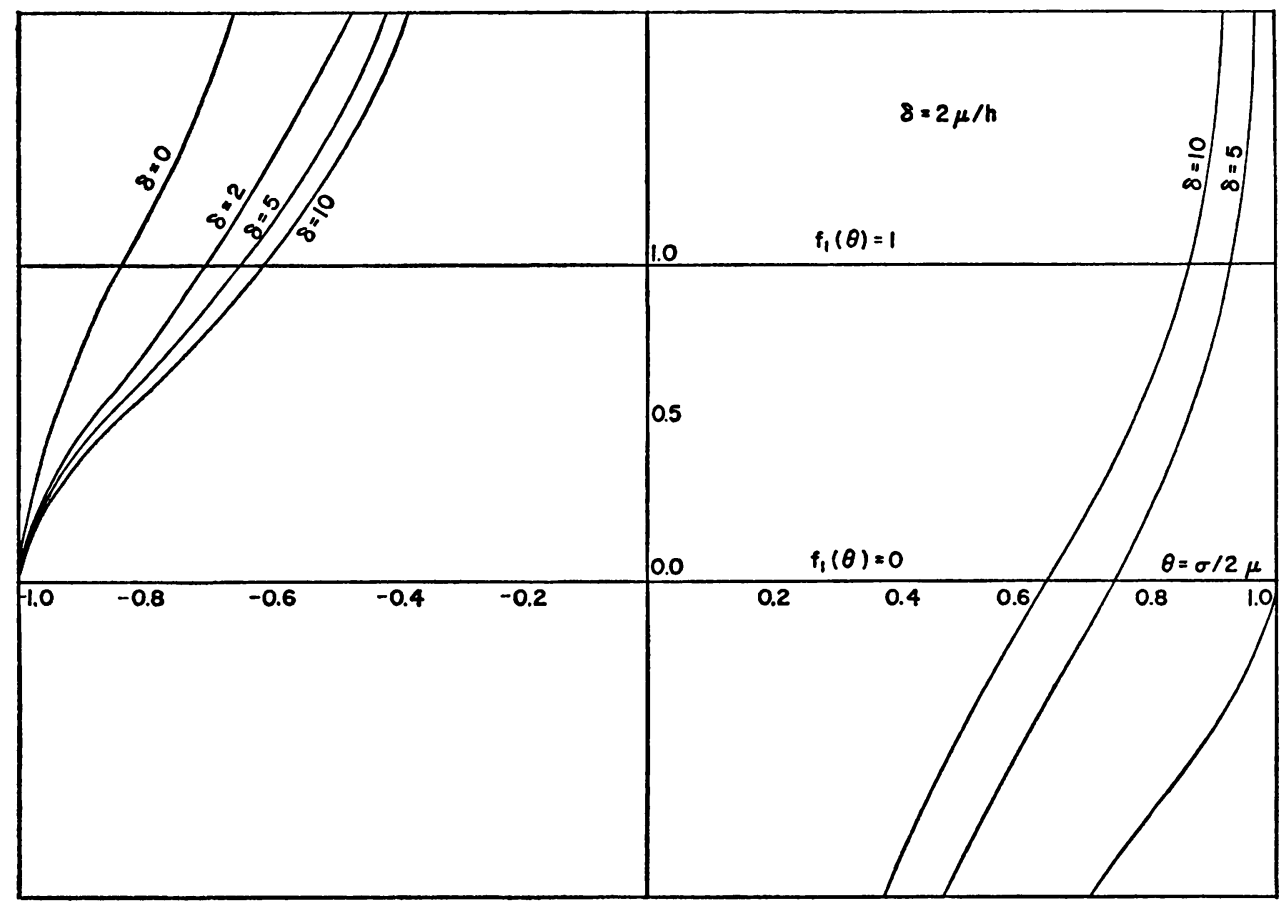

FIG. 2

It is to be noted that the case (b) is applicable also in the case of a body having a finite $c / a$ ratio and in which bifurcation is accompanied by the formation of a large number of waves.

Antisymmetric mode of deformation. The velocity field in this mode is characterized by $u(z)=-u(-z), v(z)=-v(-z), w(z)=w(-z)$. Hence, considering only the odd functions of $z$ in the expressions for $u$ and $v$,

$$
\begin{aligned}
& u=\sin \nu x \cos \eta y\left[\alpha_{1} \sinh \gamma z+\alpha_{2} \sinh \gamma \delta_{1} z+\alpha_{3} \sinh \gamma \delta_{2} z\right], \\
& v=\cos \nu x \sin \eta y\left[(-\nu / \eta) \alpha_{1} \sinh \gamma z+(\eta / \nu)\left(\alpha_{2} \sinh \gamma \delta_{2} z+\alpha_{3} \sinh \gamma \delta_{2} z\right)\right], \\
& w=-\gamma((1-\theta) / \nu) \cos \nu v \cos \eta y\left[\left(\alpha_{2} / \delta_{1}\right) \cosh \gamma \delta_{1} z+\left(\alpha_{3} / \delta_{2}\right) \cosh \gamma \delta_{2} z\right] .
\end{aligned}
$$

By a procedure similar to that employed for the case of symmetric mode of deformation, the following condition for the existence of nontrivial solutions for $\alpha_{1}, \alpha_{2}$ and $\alpha_{3}$ is obtained:

$$
\frac{\delta_{2} \tanh \gamma \delta_{2} c}{\delta_{1} \tanh \gamma \delta_{1} c}=\frac{\left[\frac{3}{1+\delta}-\theta-\delta_{1}^{2}\right]\left(1-\theta+\delta_{2}^{2}\right)}{\left[\frac{3}{1+\delta}-\theta-\delta_{2}^{2}\right]\left(1-\theta+\delta_{1}^{2}\right)}
$$

For a thin plate for which $c / a, c / b \ll 1$, the critical stress is found to be 


$$
\begin{aligned}
\sigma & =-E_{t} \frac{\pi^{2} c^{2}}{3}\left(\frac{m^{2}}{a^{2}}+\frac{n^{2}}{b^{2}}\right)\left[1+\frac{\pi^{2} c^{2}}{15} \frac{\delta-17}{\delta+1}\left(\frac{m^{2}}{a^{2}}+\frac{n^{2}}{b^{2}}\right)\right], \\
E_{t} & =\mu \frac{4+\delta}{1+\delta}
\end{aligned}
$$

The minimum value of $\sigma$ in (24) corresponds to $m=1, n=1$.

For large values of $c / a, c / b, \mathrm{Eq}$. (23) reduces to a form already discussed in case (b) under symmetric mode.

Surface instability. The disturbance at the surface in this case is assumed to decay rapidly as the depth from the surface increases. Taking the free surface to be the $x-y$ plane with the positive direction of the $z$-axis pointing into the body, the incremental field variables may be taken in the form $f(x, y) \exp (-k z), k>0$. The components of the velocity field $v_{i}$ are then found to be

$$
\begin{aligned}
& u=\sin \nu x \cos \eta y\left[\alpha_{1} \exp (-\gamma z)+\alpha_{2} \exp \left(-\gamma \delta_{1} z\right)+\alpha_{3} \exp \left(-\gamma \delta_{2} z\right)\right], \\
& v=\cos \nu x \sin \eta y\left[\alpha_{1}(-\nu / \eta) \exp (-\gamma z)+(\eta / \nu) \alpha_{2} \exp \left(-\gamma \delta_{1} z\right)+\alpha_{3} \exp \left(-\gamma \delta_{2} z\right)\right], \\
& w=\gamma((1-\theta) / \nu) \cos \nu x \cos \eta y\left[\left(\alpha_{2} / \delta_{1}\right) \exp \left(-\gamma \delta_{1} z\right)+\left(\alpha_{3} / \delta_{2}\right) \exp \left(-\gamma \delta_{2} z\right)\right] .
\end{aligned}
$$

The surface $z=0$ is considered to be free of surface traction-rate. Hence from the boundary condition (2c), using (11), follows

$$
\dot{\sigma}_{i j}-\sigma_{i k} v_{3, k}=0 \quad(j=1,2,3) \text { at } z=0 .
$$

The equations (9), (10) and (25) can be used to express (2d) in the following form:

$$
\begin{aligned}
& \quad \alpha_{1}+\alpha_{2}\left[\delta_{1}+(1-\theta) / \delta_{1}\right]+\alpha_{3}\left[\delta_{2}+(1-\theta) / \delta_{2}\right]=0, \\
& -\alpha_{1}+\alpha_{2}\left(\eta^{2} / \nu\right)\left[\delta_{1}+(1-\theta) / \delta_{1}\right]+\alpha_{3}\left(\eta^{2} / \nu^{2}\right)\left[\delta_{2}+(1-\theta) / \delta_{2}\right]=0, \\
& \quad \alpha_{2}\left[\frac{3}{1+\delta}-\theta-\delta_{1}^{2}\right]+\alpha_{3}\left[\frac{3}{1+\delta}-\theta-\delta_{2}^{2}\right]=0 .
\end{aligned}
$$

A necessary and sufficient condition for existence of nontrivial solutions for $\alpha_{1}, \alpha_{2}$ and $\alpha_{3}$ is

$$
\left(\frac{1+\theta}{1-\theta}\right)^{1 / 2}\left[1-\frac{4+\delta}{2 \theta(1+\delta)}\right]=1 .
$$

This equation has already been discussed in case (b) under symmetric mode. It may be noted that the mode of deformation localized at the surface is possible under both tensile and compressive loads. For a body with finite $c / a$ and $c / b$ ratio, the local instability is characterized by the formation of large numbers of waves on the surface.

For a semi-infinite elastic solid, (26) yields $\theta^{3}-2 \theta^{2}+2=0$, which has single real root $\sigma=-1.68 \mu$.

Loading criterion. The criterion for plastic loading is $m_{i j}\left(D \sigma_{i j} / D t\right)>0$ which, with the help of (5) and (6), may be written $m_{i j} \epsilon_{i j}>0$. Substituting from (3a), (14) and (20), the loading criterion for the symmetric mode of deformation yields

$$
\frac{U}{a}+\frac{V}{b}-\eta_{3} \cos \nu x \cos \eta y\left(\alpha_{2} \cosh \gamma \delta_{1} c+\alpha_{3} \cosh \gamma \delta_{2} c\right) \gg 0 .
$$


Hence the material will load everywhere if

$$
\alpha_{2} \cosh \gamma \delta_{1} c+\alpha_{3} \cosh \gamma \delta_{2} c \leq \frac{U}{a}+\frac{V}{b} .
$$

Similarly, for antisymmetric mode of deformation, the loading criterion is obtained in the form

$$
\alpha_{2} \sinh \gamma \delta_{1} c+\alpha_{3} \sinh \gamma \delta_{2} c \leq \frac{U}{a}+\frac{V}{b}
$$

\section{REFERENCES}

[1] G. R. Cowper and E. T. Onat, The initiation of necking in plane plastic flow, Proc. 4th U. S. Nat. Cong. Appl. Mech., 1023-1029 (1962)

[2] E. T. Onat and W. Prager, The necking of a tension specimen in plane plastic flow, J. Appl. Phys. 25, 4, 491-493 (1954)

[3] T. Y. Thomas, Plastic flow and fracture in solids, Academic Press, New York, 1961

[4] S. T. Ariaratnam and R. N. Dubey, Some cases of bifurcation in elastic-plastic solids in plane strain, Quart. Appl. Math. 27, 349-358 (1969)

[5] W. Prager, Three-dimensional plastic flow under uniform stress, Rev. Fac. Sci., Istanbul Univ., 19, 1, 23-26 (1954)

[6] R. Hill, A general theory of uniqueness and stability in elastic-plastic solid, J. Mech. Phys. Solids, 6, 236-269 (1958)

[7] R. Hill, Some basic principles in the mechanics of solids without a natural time, J. Mech. Phys. Solids 7, 209-225 (1959) 